\title{
A Taguchi approach for optimization of mass transfer coefficient in metronidazole drug delivery process and activated carbon as a carrier
}

\author{
Shahad F. Hameed*, Salih Rushdi** and Zainab T. Al-Sharify*** \\ *,** Chemical Eng. Dep. College of Engineering, University of Al-Qadisiyah, Diwaniya, Iraq \\ *** Environmental Engineering Department, College of Engineering, Mustansiriyah University. Baghdad, Iraq \\ * Corresponding Author : shahadfaiz216@gmail.com
}

$\begin{array}{ll}\text { Submitted } & : 30 / 04 / 2020 \\ \text { Revised } & : 19 / 01 / 2021 \\ \text { Accepted } & : 16 / 02 / 2021\end{array}$

\begin{abstract}
New strategies have been developed in the drug delivery system in recent years for applications like pharmacokinetics control, pharmacodynamics, undetermined toxicity, immunity, biophysics, and drug efficacy. The loading process was based on adsorption between activated carbon molecules' surfaces and drug molecules dissolved in ethanol at room temperature, where porous activated carbon has great drug delivery characteristics. The current research is studying the effect of the number of parameters including particle size, the weight of drug to the carrier, weight ratio, drug loading and temperature, time, and $\mathrm{pH}$ solution on mass transfer coefficient. The Taguchi program's result shows that the optimum point of maximum loading efficiency is $74 \%$ when the activated carbon in nanoparticle was in $11.042 \mathrm{~nm}$ size, and $985.6013 \mathrm{~m} 2 / \mathrm{g}$ surface area weight drug to AC weight ratio is 1.5 . The drug process release obtained an optimum point that gives a better value of mass transfer coefficient of 0.0007777 and 0.0003372 $\mathrm{cm} / \mathrm{hr}$ in the first hour, $37^{\circ} \mathrm{C}$, and $\mathrm{pH}$ of 1.5 solutions for both metronidazole/macro AC and metronidazole/Nano AC complexes.
\end{abstract}

Keywords: Taguchi; Nanoparticle; Drug; Mass transfer; Drug efficacy; Activated carbon.

\section{INTRODUCTION}

As the population continues to grow, the use and need for prescription drugs (antibiotics, over the counter (OTC), analgesics, etc.) will also increase (Lobaina Mato, 2019). Additionally, human and veterinary applications are the main sources of pharmaceuticals in the environment due to metabolic excretion, improper disposal, or industrial use. Consequently, most municipal sewage contains pharmaceuticals (Daughton, 2008). Unfortunately, studies have shown that most of the water treatment plants (WTPs) are insufficient and cannot eliminate many of these contaminants (Ali and Jain, 1998, Ali and Aboul-Enein, 2002, Ali et al., 2005, Basheer, 2018, Basheer, 2018, Basheer and Ali, 2018, Ali et al., 2019). Most consumers dispose of their unused and expired prescriptions by throwing them in the toilet, sink, or trash sink (Gaffney et al., 2014, Rzaij et al., 2020). 
The presence of these products and byproducts in the environment has raised concerns, such as impairment of reproductive, physiological processes, and the creation of antibiotic-resistant bacteria. Therefore, better means for effective disposal are needed and required as the human population keeps growing (Leung et al., 2013). The development of a new drug is an enormous step that consumes money, time, and effort, which increases the difficulty in medical research. The priority of any research center in the pharmaceutical industry is to reduce the overall costs and increase its productivity. A widespread drug route administration type is the oral drug or (oral route) due to its simplicity, the less patient compliance, and the cheap cost (Krumme, 2016). However, the oral drug problem is that the absorption rate can be increased or decreased depending on different factors before reaching the site of action the drug degradation.

To reach the drug's beneficial action, the administration requires sundry doses along the day with the hazard of forgetting a dose by patients. Also, the variation of the drug concentration in the plasma can be considered during the day (Downie, 2013). For example, the treatment with antibiotics or other drugs that require extended time for treatment could face the mentioned problems. One solution is to use the controlled drug delivery systems used to an embodiment of a high drug concentration to achieve the desired profile of drug release throughout the day. Different delivery systems can be controlled to modify the drug delivery process, such as immediate-release, delayed drug delivery, site-specific, and sustained drug delivery systems. In the first two types (quick and delayed drug delivery), the central system's drug release profile is accelerated, while it retarded in the delayed case (Siepmann and Siepmann, 2012).

The controlled drug delivery systems provide drug release from the dosage form through several minutes or hours. Increasing the amount of time to absorb the drug also increases drug efficacy and effectiveness while reducing the drug side effects. The downside of developing controlled drug delivery systems is the high costs, since it requires expensive equipment and particular carriers. The drug release mechanisms can illustrate by penetration the fluid to the system, the dissolution of the drug in fluid, and the drug diffuses outside the carrier matrix. These mechanism steps can occur, but in different rate and order, and the level which has the lowest rate is the controlling step or ratelimiting step and thus can control the drug release.

Adsorption occurs due to an imbalance of the molecular forces of liquids and solids' surfaces, and there are two types of adsorption, physical and chemical (Herbert et al., 2002, Al-Moameri et al., 2020, Al-sharify et al., 2020). Here, the loading process depends on physical adsorption (van der Waals adsorption). The working force between the surfaces adsorbent and absorbed is the forces of van der Waals.

$$
\text { loading eff. }=\text { (weight of the drug in a complex }) /(\text { total weight of complex })
$$

The change in drug concentration over time relies upon three factors: the diffusion of the drug into or out of the system, the amount of drug leaving or entering the system due to bulk fluid flow, and the generation or consumption of the drug within the system. The diffusion of a drug is proportional to the concentration gradient. When there is a steep concentration gradient, such as when a drug delivery carrier is placing in a fluid containing no drug, the diffusion occurs faster than when the concentration gradient is low, such as when the concentration outside of a delivery vehicle is near the concentration inside the delivery vehicle (Rossi et al., 2016, Al-Sharify et al., 2020). When concentrations in one area are equal to concentrations in an adjacent area, there will be no diffusive exchange of the molecule between those areas. Diffusion is also a function of a physical constant. The diffusion coefficient that describes the material's inherent properties is diffusing in a dense material, such as bone. The diffusion coefficient of a molecule is lower than in a free liquid such as water. Diffusion coefficients are related, among other factors, to the size of the molecule diffusing. Large molecules will diffuse more slowly and have a lower diffusion coefficient than smaller molecules (Foy and Blake, 2001, Lahieb Faisal et al., 2020). According to the Brunner and Nernst equation, the drug's disintegration in a medium is influenced by several factors such as solubility, wettability of the drug, and the dissolved 
medium's absorption conditions' surface area the drug (Banker et al., 2012). Several strategies have been developed to modify the above factors to improve the solubility or solubility rate of drugs with poor water solubility; however, such techniques often relate to limitations concerning poor stability and low drug loading. One of the many soluble enhancing techniques involves converting the crystalline drug into an amorphous state. The amorphous form's decomposition rate is significantly better than the crystalline form, especially in high energy crystalline drugs (Salonen et al., 2008). The dissolution of solid drug suspended in solution is known to be controlled with time by the diffusion of this drug away from the solid surface of the undissolved material until reaching saturation concentration (Cussler, 2009). Start with a mass balance equation to associate the dissolution result with a mass transfer coefficient, then integrate it with boundary condition $\mathrm{C}=0$ at $\mathrm{t}=0$.

$$
\text { accumulation of drug in solution }=\text { total dissolution rate }
$$

$$
V d c / d t=A j \quad j=A K\left(c_{s a t}-c\right)
$$

Supequation (3) in (2)

$$
V \frac{d c}{d t}=A K\left(c_{s a t}-c\right)
$$

Rearrange eq (4)

$$
\frac{d c}{c_{s a t}-c}=\frac{A K}{V} d t
$$

Integrate eq (5) with boundary condition $\mathrm{c}=0$ at $\mathrm{t}=0$

$$
-\ln \left(c_{s a t}-c\right)=\frac{A K}{V} t+B
$$

Compensate B.C., where B is an integration constant

$B=-\ln c_{s a t}$

Sub in equation (6)

$$
\begin{gathered}
\ln \frac{c_{s a t}}{c_{\text {sat }}-c}=\frac{A K}{V} t \quad \text { or } \quad \frac{c}{c_{\text {sat }}}=1-\exp \left(-\frac{A K}{V} t\right) \\
\therefore K=\frac{V}{A t} \ln \frac{c_{\text {sat }}}{c_{\text {sat }}-c}
\end{gathered}
$$

Miriyala (Miriyala et al., 2017) studied the activated carbon used as a drug carrier, PA, and IBU as types of drugs use, loaded into AC, with a $44.4 \%$ and $20.7 \%$ loading efficiency for two drugs. The Paracetamol drug was completely amorphous and loaded onto activated carbon, while $19 \%$ of the ibuprofen drug loaded on AC was crystalline. Suggesting that the actual efficiency of loading of the amorphous ibuprofen was only $39 \%$. The study of release suggested that, within $10 \mathrm{~min}$, the drug release for two IBU/AC and PA/AC samples was completed in the presence of sodium phosphate buffer at $\mathrm{pH}$ 5.8. The current research provides a novel method for studying the effect 
of the number of parameters including particle size, the weight of drug to the carrier, weight ratio, drug loading and temperature, time, and $\mathrm{pH}$ solution on mass transfer coefficient.

\section{EXPERIMENTAL WORK}

The experimental side goes through two stages. The first stage is to get the drugs loaded on the activated carbon molecules through the loading process. The second stage is the drugs' release process, in other words, the drug's release from the AC surface. The mass transfer coefficient of the drugs was studied in the second stage. In this work, the experiments were designed by the Taguchi program as the number of two variables particle size of activated carbon (1 (nanoparticle size) and 2 (macroparticle size) and the ratio of the weight of both drug and AC as shown in Table 1.

Table 1. Taguchi design factors of the first stage.

\begin{tabular}{|c|c|c|c|c|}
\hline Factors & Name & Level values & Column & Level \\
\hline A & Size AC & 12 & 1 & 2 \\
\hline B & W.D/W.AC & 0.511 .5 & 2 & 3 \\
\hline
\end{tabular}

The procedure of the work includes dissolving Metronidazole in ethanol according to saturation solubility of Metronidazole (5mg/ml) (COMISSION 2009) as shown in Table 3. Then, $1 \mathrm{~g}$ of activated carbon was added to the solution during mixing for 24 hours by the magnetic stirrer at room temperature. After that, the solution is filtered using Buchner funnel. Later, the remaining material is dried in the oven for 4 hours at $40^{\circ} \mathrm{C}$ (Miriyala et al., 2017, Hameed and Rushdi, 2019), and the solution size will be measured, and drug concentration in solution measured by UV spectrometer. This concentration will be multiplied by the volume of the solution to determine the drug's weight unloaded and finally calculate the drug loading efficiency by used eq (1). The release of drugs was measured in two mediums PH 1.5 and $\mathrm{PH}$ 6.5. Therefore, an acid solution with a $\mathrm{pH}$ of 1.5 was prepared from $99 \%$ water injected and $1 \%$ hydrochloric acid, and the second solutions were made from $99 \%$ water injected and 1\% methanol. $50 \mathrm{mg}$ of Metronidazole was added to $100 \mathrm{ml}$ from these solutions, put the mixture in the shaker water bath, and set at three temperatures $35,37,39^{\circ} \mathrm{C}$, and $50 \mathrm{rpm}$. Then, measure the concentration at different times. In this work, the experiments were designed by Taguchi (licensing 17.1.0 2013). Three factors were considered: temperature, $\mathrm{pH}$ of the solution, and time. The $\mathrm{pH}$ of the solution was studied at two levels (1.5 and 6.5), the temperature at three levels $\left(35,37\right.$, and $\left.39^{\circ} \mathrm{C}\right)$, and time at three levels $(1,3$, and $5 \mathrm{hr})$. The mass transfer coefficient is calculated by using eq (7), as shown in Table 2. The concentration of drugs is measured at a different time, as mentioned in the above procedure and at three different temperatures $(35,37$, and 39$){ }^{\circ} \mathrm{C}$. 
Table 2. Taguchi design factors of the second stage.

\begin{tabular}{|c|c|c|c|c|}
\hline Factors & Name & Level values & Column & Level \\
\hline $\mathrm{A}$ & $\mathrm{PH}$ & 1.56 .5 & 1 & 2 \\
\hline $\mathrm{B}$ & $\mathrm{TIME}(\mathrm{hr})$ & 246 & 2 & 3 \\
\hline $\mathrm{C}$ & TEMP $\left({ }^{\circ} \mathrm{C}\right)$ & 353739 & 3 & 3 \\
\hline
\end{tabular}

\section{RESULTS AND DISCUSSION}

Drugs were loaded into blank AC, both macro- and nanoparticle size, using the method described below. Both AC purchased macro- and nanoparticle size were confirmed to be porous, as indicated in the BET full report in Appendix A. These result in $0.6 \mu \mathrm{m}$ particles size with $544.4704 \mathrm{~m} 2 / \mathrm{g}$ surface area and $11.042 \mathrm{~nm} 985.6013 \mathrm{~m} 2 / \mathrm{g}$ surface area. From the results of Table 3, there is little difference between the quantities produced and the actual quantities used. This difference is because of the mixture in the equipment used during the experiment, including the filter papers (Miriyala et al., 2017).

Since loading is carried out in aqueous conditions, drugs limited solubility in solutions restricts the amount, which may dissolve in the reaction solution. As a result, the concentrations of these drugs are greater than the saturated solubility, and the loading process becomes sterile unless the reaction is heated. Loading efficiency reaches a maximum of around 24 hours of incubation, after which the loading efficiency no longer increases and, in some cases, is even lowered.

Table 3. Calculation of loading efficiency.

\begin{tabular}{|c|c|c|c|c|c|c|c|c|c|c|}
\hline 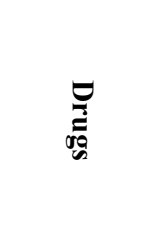 & 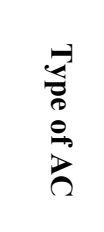 & 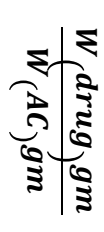 & 金兽 & 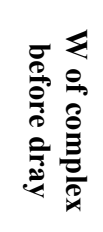 & 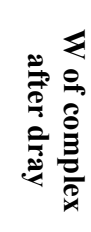 & 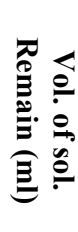 & 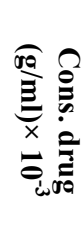 & 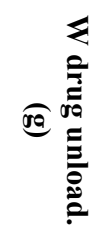 & 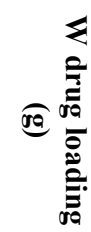 & $\underset{0}{\stackrel{2}{*}}$ \\
\hline \multirow{6}{*}{ Metronid. } & \multirow{3}{*}{ Macro } & $0.5 / 1$ & 100 & 2.241 & 0.992 & 48 & 3.73 & 0.179 & 0.32 & 32 \\
\hline & & $1 / 1$ & 200 & 2.594 & 1.136 & 125 & 3.41 & 0.42 & 0.57 & 50 \\
\hline & & $1.5 / 1$ & 300 & 2.777 & 1.309 & 210 & 2.67 & 0.561 & 0.937 & 71 \\
\hline & \multirow{3}{*}{ Nano } & $0.5 / 1$ & 100 & 3.346 & 1.12 & 41 & 3.8 & 0.155 & 0.344 & 30 \\
\hline & & $1 / 1$ & 200 & 4.099 & 1.203 & 87 & 3.5 & 0.305 & 0.695 & 57 \\
\hline & & $1.5 / 1$ & 300 & 3.441 & 1.466 & 130 & 3.2 & 0.416 & 1.084 & 74 \\
\hline
\end{tabular}




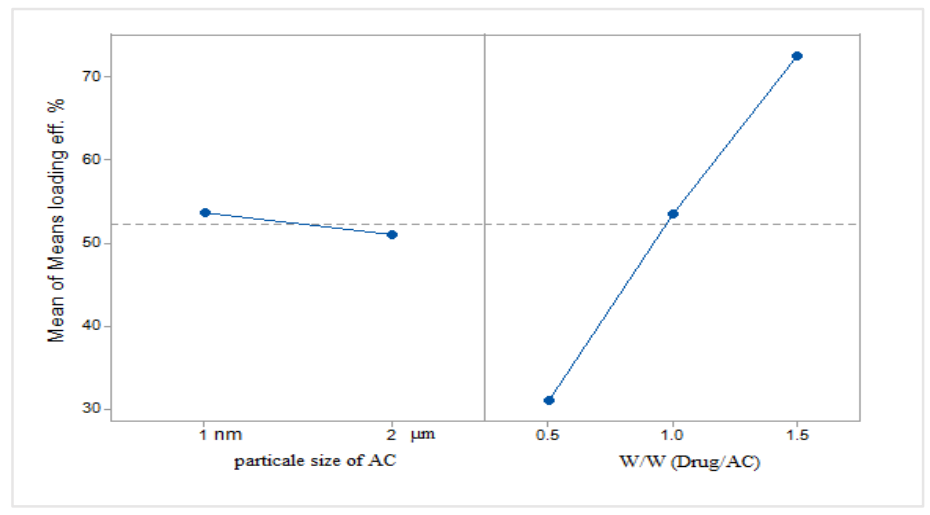

Figure 1. The optimum point of loading efficiency for Metronidazole with two factors in the Taguchi program.

The means for both particle size and drug ratio were calculated for all experiments using the Taguchi technique. The result in Figure 1 shows an increase in the drug efficiency during drug weight to AC weight ratio, which increases with the decrease in the AC particle size.

The release of the drug from the complex may be incomplete, this could be due to poor wettability of carbon particles, which may increase or decrease the emission period, and this can be due to the stronger effect of AC porosity on solubility profiles compared with the properties of the drug (Miriyala et al., 2017). The higher surface area of the $\mathrm{AC}$ pores had them to be in contact with the media solution, leading to control release; thus, the drug was loaded into nano AC, compared to pure drugs, preferably more control release also when it is compared between the macro $\mathrm{AC} /$ drug and nano AC/drug samples. Figure 2 shows the case when $\mathrm{pH} 6.5$ at 6 hours $\sim 100 \%$ drug release is pure without carbon, and $72 \%$ release from drug/macro activated carbon complex, while the best result release $62 \%$ is from drug/nano activated carbon complex, and it shows that the release is controlled at drug/nano AC complex, and when $\mathrm{pH} 1.5$ shows at 6 hours $\sim 100 \%$ drug release when the drug pure without carbon and $74 \%$ release from drug/macro activated carbon complex, while the best result release, $65 \%$, is from drug/nano activated carbon complex. This result has shown that the release of Metronidazole was more control with the loading process, and more control when activated carbon in nanoparticle size compared with macroparticle size at $37^{\circ} \mathrm{C}$ (Rushdi and Hameed, 2020).

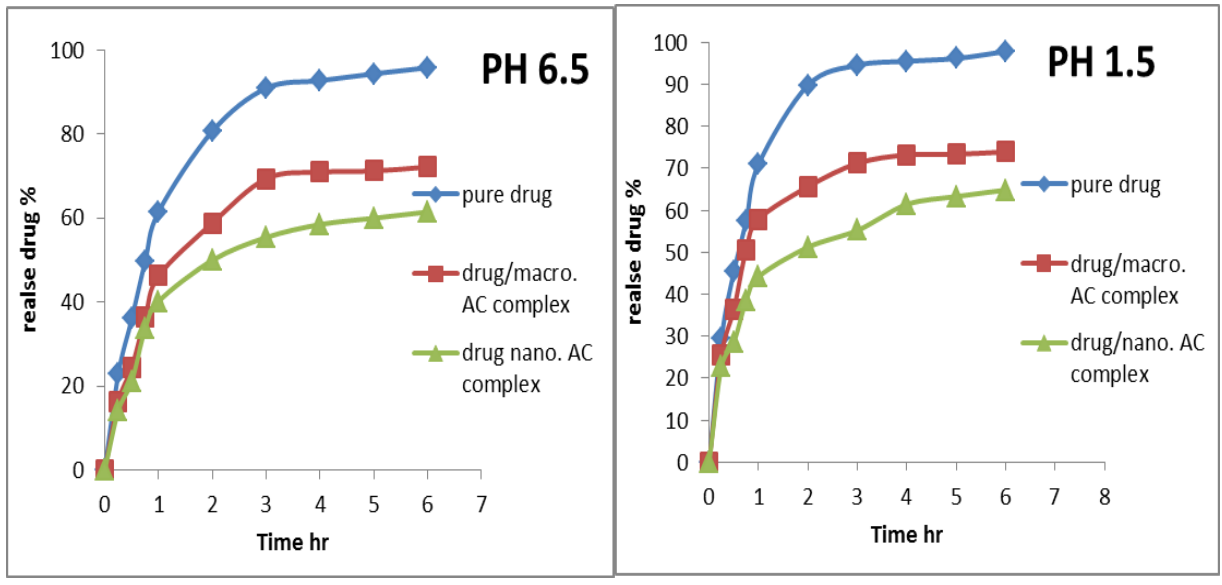

Figure 2. Metronidazole release pattern in PH 1.5 and PH 6.5 solutions. 
The unloading process behavior corresponds to researchers' work (Miriyala et al., 2017), who used activated carbon as a carrier for two types of drugs, paracetamol and ibuprofen. The microparticles of Mesoporous silicon were used as carriers to five types of drugs. The drugs were ibuprofen, ranitidine, furosemide, griseofulvin, and antipyrine. In this sense, all research in drug loading concluded that the release of the drugs from carriers had been more controlled during the time, and it takes a long time more than in case of the drug is pure.

It is noted from Figure 3 that there is some similarity in the behavior of the rate of mass transfer coefficient. The coefficient increases in the dissolution medium from zero to the highest value. Then, it begins to decline until it reaches a stage that is gradually controlled over time. The behavior demonstrates that drug diffusion is proportional to the concentration gradient. In other words, the diffusion occurs faster when the drug delivery is placed in a liquid without the drug. This conclusion leads to the fact that when the concentrations are equal in two neighboring areas, the molecular drug diffusion begins to decrease (Rossi et al., 2016).

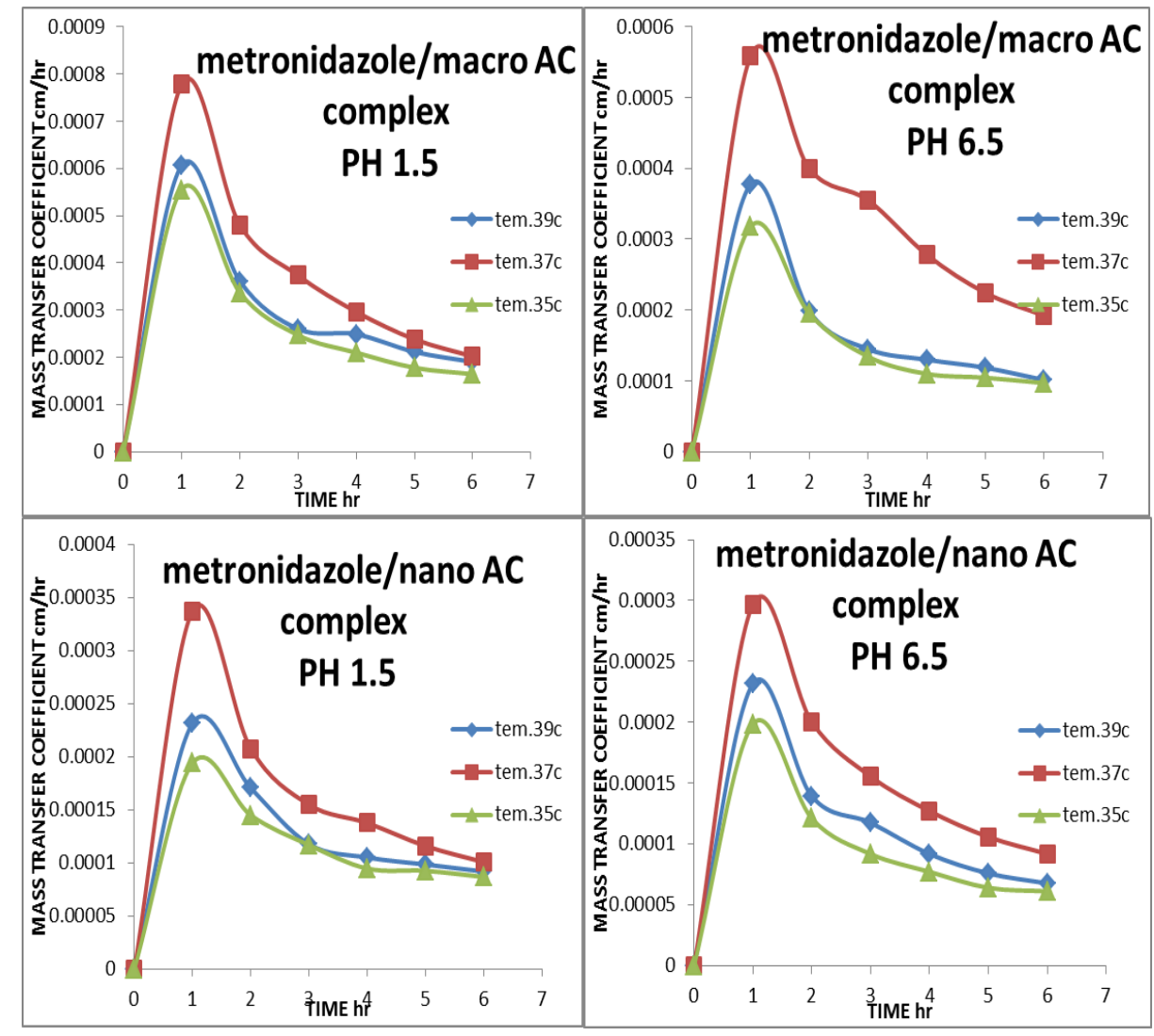

Figure 3. Mass transfer coefficient variation with time for Metronidazole.

After synthesis, drug loading, surface coupling, and nanoparticle characterization, drug release studies are conducted to study the release rate of the drug loaded into the environment by measuring the mass transfer factor. The analysis in the Taguchi design program was designed based on three factors ( $\mathrm{PH}$ solution two results (1.5 and $6.5)$, the temperature in co three levels $\left(35,37\right.$, and $\left.39^{\circ} \mathrm{C}\right)$, and time in hour three levels $(1,3$, and 5)). The results shown in Table 4 and Table 5 were analyzed to obtain the optimum points of three factors on the mass transfer coefficient. 
Regression Equations for Mass Transfer Coefficient multiple Linear Regression Model are $y=b 0+b 1 \times 1 i+b 2 \times 2 i+\cdots+b k x k i$

where $\mathrm{y}$ is the observed response (mass transfer coefficient $\mathrm{cm} / \mathrm{hr}$ ) to the values $\mathrm{x} 1 \mathrm{i}, \mathrm{x} 2 \mathrm{i}, \ldots$, xki of the $\mathrm{k}$ independent variables $\mathrm{x} 1, \mathrm{x} 2, \ldots, \mathrm{xk}$ : three factors (X1 tem., X2 time, and X3 PH solution), i number of levels (three levels for $\mathrm{X} 1$ and $\mathrm{X} 2\left(35^{\circ} \mathrm{C}, 37^{\circ} \mathrm{C}, 39^{\circ} \mathrm{C}, 1 \mathrm{hr}, 3 \mathrm{hrs}\right.$ and $\left.5 \mathrm{hrs}\right)$ sequentially and two levels for $\mathrm{X} 3(1.5$ and 6.5$\left.)\right)$ as shown in Table 6. The main effect plots analysis shown in Figure 4 and Figure 5. There is no significant effect if the line was horizontal in the sense that all levels of factors on response value were in the same way.

Table 4. Experimental design and results for metronidazole/ macro AC complex.

\begin{tabular}{|c|c|c|c|c|c|c|}
\hline No. & $\mathbf{p H}$ & Time (hr) & Temp. $(\mathbf{C})$ & M.T.C (cm/hr) & SNRA1 & MEAN1 \\
\hline 1 & 1.5 & 1 & 35 & 0.0005550 & -65.1137 & 0.0005550 \\
\hline 2 & 1.5 & 1 & 37 & 0.0007777 & -62.1835 & 0.0007777 \\
\hline 3 & 1.5 & 1 & 39 & 0.0006057 & -64.3542 & 0.0006057 \\
\hline 4 & 1.5 & 3 & 35 & 0.0002469 & -72.1498 & 0.0002469 \\
\hline 5 & 1.5 & 3 & 37 & 0.0003734 & -68.5575 & 0.0003734 \\
\hline 6 & 1.5 & 3 & 39 & 0.0002600 & -71.7011 & 0.0002600 \\
\hline 7 & 1.5 & 5 & 35 & 0.0001778 & -75.0027 & 0.0001778 \\
\hline 8 & 1.5 & 5 & 37 & 0.0002381 & -72.4651 & 0.0002381 \\
\hline 9 & 1.5 & 5 & 39 & 0.0002114 & -73.4961 & 0.0002114 \\
\hline 10 & 6.5 & 1 & 35 & 0.0003181 & -69.9495 & 0.0003181 \\
\hline 11 & 6.5 & 1 & 37 & 0.0005588 & -65.0549 & 0.0005588 \\
\hline 12 & 6.5 & 1 & 39 & 0.0003777 & -68.4573 & 0.0003777 \\
\hline 13 & 6.5 & 3 & 35 & 0.0001342 & -77.4457 & 0.0001342 \\
\hline 14 & 6.5 & 3 & 37 & 0.0003552 & -68.9913 & 0.0003552 \\
\hline 15 & 6.5 & 3 & 39 & 0.0001449 & -76.7805 & 0.0001449 \\
\hline 16 & 6.5 & 5 & 35 & 0.0001044 & -79.6242 & 0.0001044 \\
\hline 17 & 6.5 & 5 & 37 & 0.0002242 & -72.9855 & 0.0002242 \\
\hline 18 & 6.5 & 5 & 39 & 0.0001185 & -78.5270 & 0.0001185 \\
\hline
\end{tabular}


Table 5. Experimental design and results for metronidazole/Nano AC complex.

\begin{tabular}{|c|c|c|c|c|c|c|}
\hline No. & pH & Time (hr) & Temp. ( $\left.{ }^{\circ} \mathrm{C}\right)$ & M.T.C (cm/hr) & SNRA1 & MEAN1 \\
\hline 1 & 1.5 & 1 & 35 & 0.0001939 & -74.2483 & 0.0001939 \\
\hline 2 & 1.5 & 1 & 37 & 0.0003372 & -69.4411 & 0.0003372 \\
\hline 3 & 1.5 & 1 & 39 & 0.0002315 & -72.7103 & 0.0002315 \\
\hline 4 & 1.5 & 3 & 35 & 0.0001169 & -78.6472 & 0.0001169 \\
\hline 5 & 1.5 & 3 & 37 & 0.0001548 & -76.2044 & 0.0001548 \\
\hline 6 & 1.5 & 3 & 39 & 0.0001175 & -78.5980 & 0.0001175 \\
\hline 7 & 1.5 & 5 & 35 & 0.0000925 & -80.6766 & 0.0000925 \\
\hline 8 & 1.5 & 5 & 37 & 0.0001159 & -78.7187 & 0.0001159 \\
\hline 9 & 1.5 & 5 & 39 & 0.0000985 & -80.1311 & 0.0000985 \\
\hline 10 & 6.5 & 1 & 35 & 0.0001986 & -74.0392 & 0.0001986 \\
\hline 11 & 6.5 & 1 & 37 & 0.0002971 & -70.5422 & 0.0002971 \\
\hline 12 & 6.5 & 1 & 39 & 0.0002317 & -72.7029 & 0.0002317 \\
\hline 13 & 6.5 & 3 & 35 & 0.0000919 & -80.7370 & 0.0000919 \\
\hline 14 & 6.5 & 3 & 37 & 0.0001556 & -76.1612 & 0.0001556 \\
\hline 15 & 6.5 & 3 & 39 & 0.0001175 & -78.5959 & 0.0001175 \\
\hline 16 & 6.5 & 5 & 35 & 0.0000641 & -83.8599 & 0.0000641 \\
\hline 17 & 6.5 & 5 & 37 & 0.0001059 & -79.5015 & 0.0001059 \\
\hline 18 & 6.5 & 5 & 39 & 0.0000760 & -82.3860 & 0.0000760 \\
\hline
\end{tabular}

Table 6. Regression equations for mass transfer coefficient.

Metronidazole /macro.AC $\mathrm{y}=0.000321-0.000065 \mathrm{X} 11+0.000100 \mathrm{X} 12-0.000035 \mathrm{X} 13+0.000211 \mathrm{X} 21-$ $0.000069 \times 22-0.000142 \times 23+0.000062 \times 31-0.000062 \times 32$
Metronidazole /nano.AC $\mathrm{y}=0.000155-0.000029 \times 11+0.000039 \times 12-0.000010 \times 13+0.000093$ $\mathrm{X} 21-0.000030 \mathrm{X} 22-0.000063 \mathrm{X} 23+0.000007 \mathrm{X} 31-0.000007 \mathrm{X} 32$ 
The average response had the same levels of factors. There would be a significant effect if the line was not horizontal, because the levels' effect differed from one factor to another. The larger variety of plots was in the vertical axis; in other words, the drawn points were not parallel to the $\mathrm{X}$-axis, the greater value of the main effect. The relative quantity of factors effect can be compared by comparing slope lines (Dar and Anuradha, 2018).

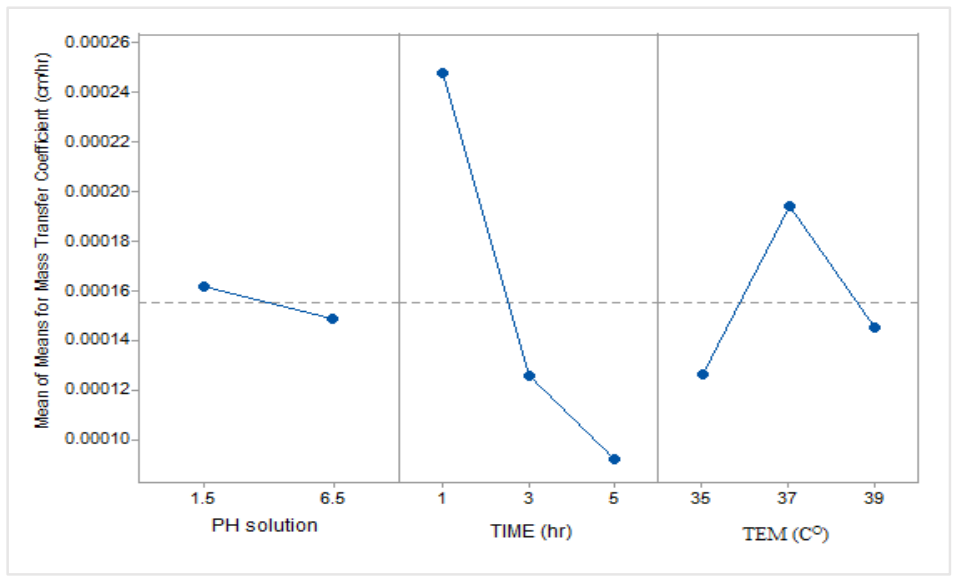

Figure 4. The optimum point of mass transfer coefficient of metronidazole Nanoparticle AC with three-factor in the Taguchi program.

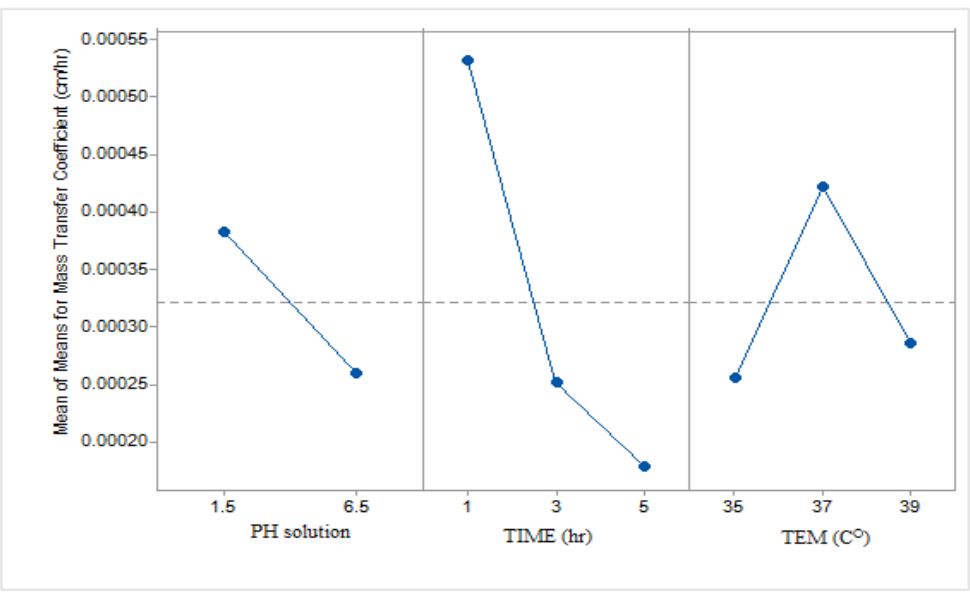

Figure 5. The optimum point of mass transfer coefficient of metronidazole macro AC particle with three factors in the Taguchi program.

A residual plot is a graph in Figures 6 and 7 that shows the residuals on the vertical axis and the independent variable on the horizontal axis. If the residual plot points are randomly dispersed around the horizontal axis, a linear regression model is appropriate for the data; otherwise, a nonlinear model is appropriate. 


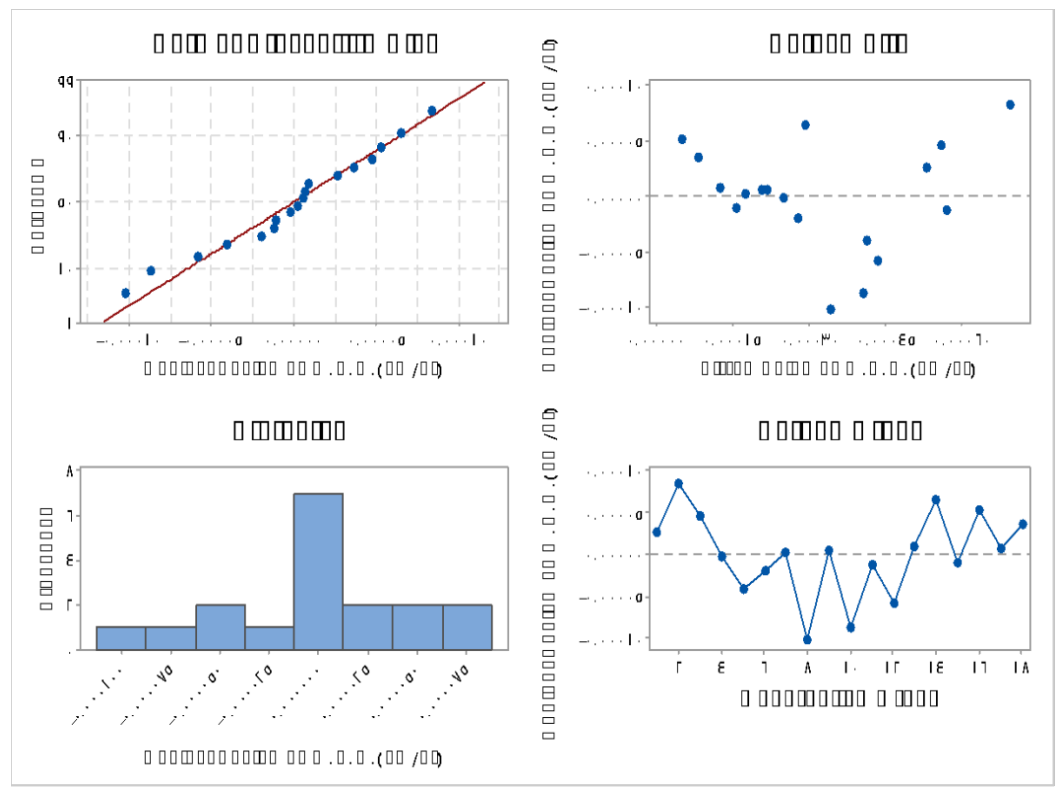

Figure 6. Residual plots for metronidazole /macro AC complex.

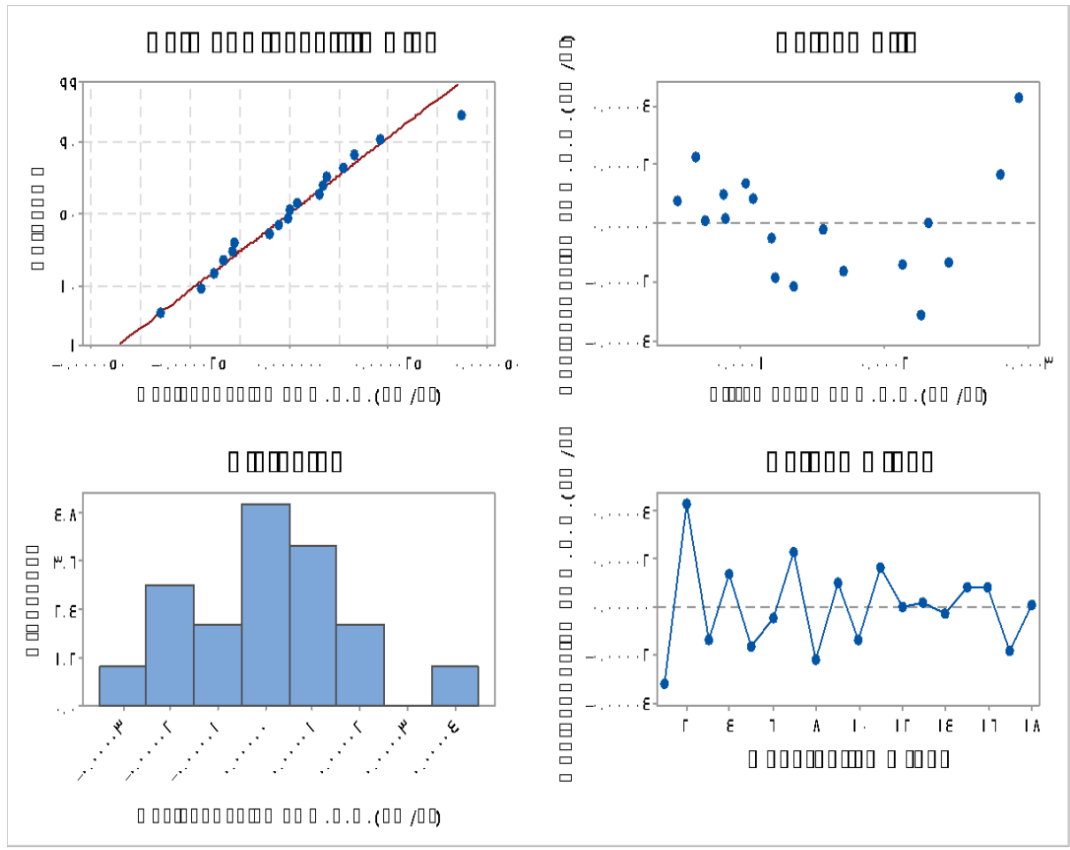

Figure 7. Residual plots for metronidazole/nano AC complex.

This random pattern indicates that a linear model provides a decent fit to the data. It indicates a good fit for a linear model, a long tail in one direction, and the pattern may indicate curvature, and A bar that is far away from the other bars may indicate an outlier. Outliers can be described as extremely low or high values that do not fall near any other data points. 


\section{CONCLUSIONS}

Metronidazole was loaded successfully into AC, with a maximum loading efficiency for Metronidazole 74\% and $71 \%$ in nano AC and macro AC, respectively. The loading efficiency of drugs increased when carriers' particle size decreased, and when the ratio of the weight of drugs to the carrier's weight was greater than one. The drug's unloading was better controlled when the activated carbon particle was in nano size, giving mass transfer control. The optimum temperature was $37^{\circ} \mathrm{C}$ to solution medium determining whether $\mathrm{AC}$ is in macro or nano size. Besides, the difference in $\mathrm{pH}$ of solution values has less effect on release drugs, all the solutions used were acidic. These correspond to the middle of the stomach and intestines of the human.

\section{ABBREVIATIONS}

A: area of the particle $\left(\mathrm{m}^{2}\right)$

AC: activated carbon

CNTs: carbon nanotubes

C1Sat: concentration of drug in the surface of the particle (saturated solubility of the drug in fluid) $\left(\mathrm{mol} / \mathrm{m}^{3}\right)$

$\mathrm{C} 1$ : concentration of drug at the edge of the fluid diffusion layer $\left(\mathrm{mole} / \mathrm{m}^{3}\right)$

D: drug

IBU: ibuprofen

$\mathrm{j} 1$ : Molar flux rate of drug $\left(\mathrm{mole} / \mathrm{m}^{2} . \mathrm{hr}\right)$

$\mathrm{K}$ : overall mass transfer coefficient $(\mathrm{m} / \mathrm{hr})$

MWCNT: multi-walled carbon nanotubes

PA: paracetamol

TEM: temperature

$\mathrm{V}$ : volume of solution $\left(\mathrm{m}^{3}\right)$

W: weight

6 MP: 6 Mercaptopurine

\section{ACKNOWLEDGMENT}

The authors would like to thank the University of Al-Qadisiyah, Diwaniya, Iraq (www.qu.edu.iq), and Mustansiriyah University (www.uomustansiriyah.edu.iq) Baghdad, Iraq, for their support in the present work.

\section{REFERENCES}

Al-Moameri, H., N. Al-sharify, M. Abdulrehman \& Z. T. Al-sharify, 2020. "Impact of Nanoparticles on Polyurethane Resin's Final Properties." Journal of Green Engineering 10: 3114-3126.

Al-sharify, N., Z. T. Al-sharify, T. A. Alsharify, M. Al-Sharify \& A. Alsharify, 2020. "A Technical Overview and Comparison between PET and MRI Scanning." Systematic Reviews in Pharmacy 11: 35-41.

Al-Sharify, Z. T., T. A. Al-Sharify, N. T. Al-Sharify \& H. Yahya naser, 2020. "A critical review on medical imaging techniques (CT and PET scans) in the medical field." IOP Conference Series: Materials Science and Engineering 870: 012043.

Ali, I. \& H. Y. Aboul-Enein, 2002. "Speciation of Metal Ions by Capillary Electrophoresis." Critical Reviews in Analytical Chemistry 32(4): 337-350.

Ali, I., O. M. L. Alharbi, A. L. ZA, A. M. Al-Mohaimeed \& A. Alwarthan, 2019. "Modeling of fenuron pesticide adsorption on CNTs for mechanistic insight and removal in water." Environ Res 170: 389-397. 
Ali, I., V. K. Gupta \& H. Y. Aboul-Enein, 2005. "Metal ion speciation and capillary electrophoresis: application in the new millennium." Electrophoresis 26(21): 3988-4002.

Ali, I. \& C. K. Jain, 1998. "Groundwater contamination and health hazards by some of the most commonly used pesticides." Current Science 75(10): 1011-1014.

Banker, G. S., J. Siepmann \& C. Rhodes, 2012. Modern Pharmaceutics. Boca Raton, CRC Press.

Basheer, A. A., 2018. "Chemical chiral pollution: Impact on the society and science and need of the regulations in the 21 st century." Chirality 30(4): 402-406.

Basheer, A. A., 2018. "New generation nano-adsorbents for the removal of emerging contaminants in water." Journal of Molecular Liquids 261: 583-593.

Basheer, A. A. \& I. Ali, 2018. "Stereoselective uptake and degradation of ( \pm )-o,p-DDD pesticide stereomers in water-sediment system." Chirality 30(9): 1088-1095.

Cussler, E. L., 2009. Diffusion: Mass Transfer in Fluid Systems. UK, Cambridge university press.

Dar, A. A. \& N. Anuradha, 2018. "Comparison: Binomial model and Black Scholes model[J]. Quantitative Finance and Economics." Quantitative Finance and Economics 2(1): 230-245.

Daughton, C., 2008. Pharmaceuticals as Environmental Pollutants: The Ramifications for Human Exposure: 66102.

Downie, P. F. k., 2013. "Extended-release drug delivery technology, Innov. Pharm. Technol." 80-86.

Foy, B. D. \& J. Blake, 2001. "Diffusion of paramagnetically labeled proteins in cartilage: enhancement of the 1D NMR imaging technique." J Magn Reson 148(1): 126-134.

Gaffney, V. d. J., V. V. Cardoso, A. Rodrigues, E. Ferreira, M. J. Benoliel \& C. M. M. Almeida, 2014. "Análise de fármacos em águas por SPE-UPLC-ESI-MS/MS." Química Nova 37: 138-149.

Hameed, S. \& S. Rushdi, 2019. "Mass Transfer Coefficient Study for Unloading of Naproxen from Activated Carbon as Drug Delivery." Al-Qadisiyah Journal for Engineering Sciences 12: 232-239.

Herbert, D. b. R., T. J. Nolan, G. A. Schad \& D. Abraham, 2002. "The role of B cells in immunity against larval Strongyloides stercoralis in mice." Parasite Immunology 24(2): 95-101.

Krumme, M., 2016. "Control strategies for continuous processes." talk at 10th World Meeting on Pharmaceutics, Biopharmaceutics and Pharmaceutical Technology, Glasgow (UK).

Lahieb Faisal, M., S. Z. Al-Najjar \& Z. T. Al-Sharify, 2020. "Modified orange peel as sorbent in removing of heavy metals from aqueous solution." Journal of Green Engineering 10(11): 10600-10615.

Leung, H. W., L. Jin, S. Wei, M. M. Tsui, B. Zhou, L. Jiao, P. C. Cheung, Y. K. Chun, M. B. Murphy \& P. K. Lam, 2013. "Pharmaceuticals in tap water: human health risk assessment and proposed monitoring framework in China." Environ Health Perspect 121(7): 839-846.

Lobaina Mato, Y., 2019. "Nasal route for vaccine and drug delivery: features and current opportunities." International Journal of Pharmaceutics: 118813.

Miriyala, P., N. P. Sukumaran, B. N. Nath, P. B. Ramamurty, A. V. Sijinkumar, B. Vijayagopal, V. Ramaswamy \& T. Sebastian, 2017. "Increased chemical weathering during the deglacial to mid-Holocene summer monsoon intensification." Scientific Reports 7(1): 44310.

Rossi, S., M. Cozzolino \& M. T. Carrì, 2016. "Old versus New Mechanisms in the Pathogenesis of ALS." Brain Pathology 26(2): 276-286.

Rushdi, S. A. \& S. F. Hameed, 2020. "Experimental Study for Loading \& Unloading of Cefixime on Activated Carbon as Drug Delivery." Systematic Reviews in Pharmacy 11(1): 40-50.

Rzaij, D. R., H. J. Al-Jaaf, S. Z. Al-Najjar, Z. T. Al-Sharify, H. H. Al-Moameri \& N. A. Mohammed, 2020. "Studying the Concentrations of Nitrite and Nitrate of Tigris River Water in Baghdad and Their Suitability 
to the Conditions Permitted Internationally." IOP Conference Series: Materials Science and Engineering 870: 012025 .

Salonen, J., A. Tötterman, J. Hirvonen \& V.-P. Lehto, 2008. "Mesoporous Silicon in Drug Delivery Applications." Journal of pharmaceutical sciences 97: 632-653.

Siepmann, J. \& F. Siepmann, 2012. "Modeling of diffusion controlled drug delivery." Journal of Controlled Release 161(2): 351-362. 